POS PROCEEDINGS

\title{
A field-theoretic approach to Spin Foam models in Quantum Gravity
}

\section{Patrizia Vitale*}

Dipartimento di Scienze Fisiche, Università di Napoli Federico II and INFN, Sezione di Napoli, Via Cintia 80126 Napoli, Italy

E-mail: vitale@na.infn.it

We present an introduction to Group Field Theory models, motivating them on the basis of their relationship with discretized BF models of gravity. We derive the Feynmann rules and compute quantum corrections in the coherent states basis.

Corfu Summer Institute on Elementary Particles and Physics - Workshop on Non Commutative Field Theory and Gravity,

September 8-12, 2010

Corfu Greece

\footnotetext{
* Speaker.
} 


\section{Introduction}

In this article we review, in a very elementary way, some of the motivations which are at the basis of a class of quantum field theories which have been proposed to describe quantum gravity $[1,2,3,4]$. The base manifold where these models are defined is represented by $D$ copies of the Lorentz group, where $D$ is the physical space-time dimension, and the Lorentz group is replaced by rotations when the signature of the metric is chosen to be Riemannian. For this reason they are known as Group Field Theories (GFT). They can also be viewed as higher rank tensor field theories, thus generalising matrix models which describe gravity in two dimensions, and sharing some features with noncommutative quantum field theories. The main motivation for us to investigate such models is that they provide a background independent formulation of quantum gravity in which one sums both over topologies and geometries. Indeed, each Feynman graph of a $D$ dimensional GFT can be dually associated with a specific space-time triangulation. Moreover, it can be seen to reproduce exactly spin-foam amplitudes [5], although in a different basis.

The simplest group field theories correspond to quantization of the BF models, hence to topological versions of gravity. The first non-topological model is the Barrett-Crane model [6]. Recently new spin foam models have been proposed for the quantization of the full $3+1$ dimensional gravity [7, 8]. These models stem from an improved analysis of the Plebanski simplicity constraints. These new theories could be called dynamical since their propagators, combining two non commuting projectors, have non-trivial spectrum. In this paper, after an introductory part dedicated to motivations and to the discretization of gravity along the lines of lattice gauge theories, we focus on such new models, derive the Feynmann rules and outline the computation of quantum corrections in the coherent states basis. A detailed account of the results may be found in [9].

The paper is organized as follows. In section 2 we review the discrete path integral derivation of $2+1$ gravity in the BF description. In section 3 we introduce the main tools of GFT, which we apply to $S O(D)$ BF theories is section 4. We compute Feynmann amplitudes of such models and we find that they coincide, for $D=3$, with the partition function of $2+1$ gravity that we have found in section 2. This serves as a motivation for the description of quantum gravity in terms of GFT models. Therefore we review in section 5 the first order formalism for the gravity action in $3+1$ dimensions in the Holst formulation and we study, in section 6, a GFT model for such an action. Finally, in section 7, we describe the stationary phase method used in [9] to evaluate the asymptotic large spin regime of certain graphs.

\section{Discrete Gravity in $2+1$ dimensions}

In this section we briefly review the first order formalism for gravity in $2+1$ dimensions and its discretization, to arrive at a discretized path integral. The partition function so obtained will be the basis to introduce and motivate GFT models, which reproduce the same result.

In $2+1$ dimensions the first order action of gravity has the form of a BF action with gauge group $G=S O(2,1)(S O(3)$ if the metric is Riemannian)

$$
S=\int \operatorname{Tr}(e \wedge F[\omega])=\int \varepsilon_{I J K} e^{I} F^{J K}[\omega]
$$


where $e$ (the B field) is a vector valued one form on the space-time manifold $M$ and $F$ the curvature of the connection one form, $\omega$, valued in the Lie algebra $\mathscr{L}=\operatorname{so}(2,1)(\operatorname{so}(3)$ in the Riemannian case). In the language of fiber bundles $\omega$ is the connection one form of the principal G-bundle, while $e$ is a one form on the associated vector bundle, it is therefore vector valued. Once we have chosen a trivialization, we can identify the fibers of the vector bundle with the Lie algebra, so that $e, \omega$ and $F$ are all $\mathscr{L}$ valued

$$
\begin{aligned}
e & =e_{\mu}^{I} d x^{\mu} \tau_{I} \\
\omega & =\omega_{\mu}^{I} d x^{\mu} \tau_{I} \\
F & =F_{\mu v}^{I} d x^{\mu} \wedge d x^{v} \tau_{I}
\end{aligned}
$$

$\tau_{I} \in \mathscr{L}$. Therefore, $\operatorname{Tr}(e \wedge F)$ is the 3 -form constructed by taking the wedge product of the differential form parts of e and $\mathrm{F}$ and using the Killing form $<.,.\rangle=-\operatorname{Tr}(.$.$) to pair their Lie algebra$ valued parts. The trace is taken in the adjoint representation.

In order to discretize this action on a lattice, let us consider a triangulation, $T$, of the spacetime manifold in terms of tetraedra, with faces $f$, edges $\ell$ and vertices $v$. We associate to the one form $e$ the Lie algebra element

$$
E_{\ell}=\int_{\ell} e
$$

To get the discrete analogue of the curvature as a quantity which is still associated to the edges we need to introduce a dual lattice, $T_{*}$. This is defined on associating to each tetraedron a vertex in the middle, $v_{*}$, to each face an edge which intersects it, $\ell_{*}$, to each edge a face $f_{*}$ whose boundary $\partial f_{*}$ encircles the edge, to each vertex a closed surface which encloses it. The two-dimensional subcomplex contained in such a cellular complex, whose building blocks are vertices, edges and faces, draws a graph which we indicate with $\mathscr{G}$, also known in the literature as dual skeleton. Therefore we associate to the connection $\omega$ the group element

$$
h_{\ell_{*}}=P \exp \int_{\ell_{*}} \omega
$$

and the discretized curvature is defined as the holonomy of the connection along the boundary of $f_{*}$. Dual faces are associated to edges of the direct triangulation, therefore we have:

$$
H_{f_{*}} \equiv H_{\ell}=\prod_{l_{*} \in \partial f_{*}(\ell)} h_{\ell_{*}}
$$

The discretized BF action becomes then

$$
S\left(E_{\ell}, H_{\ell}\right)=\sum_{\ell \in T} \operatorname{Tr} E_{\ell} H_{\ell}
$$

where the trace is to be taken in the fundamental representation of the group. We can define a discretized partition function

$$
Z\left[T, T_{*}\right]=\int_{\mathscr{L}} \prod_{\ell \in T} d E_{\ell} \int_{G} \prod_{\ell_{*} \in T_{*}} d h_{l_{*}} \exp \left(i \operatorname{Tr}\left(E_{\ell} \prod_{\ell_{*} \in \partial f_{*}} h_{\ell_{*}}\right)\right.
$$


where the Lie group $G$ is either $S O(2,1)$ or $S O(3)$, depending on the signature of the space-time manifold, while $\mathscr{L}$ is the appropriate Lie algebra. The integral in the Lie algebra can be performed and we get

$$
Z\left[T_{*}\right]=\int \prod_{\ell_{*} \in T_{*}} d h_{\ell_{*}} \prod_{f_{*} \in T_{*}} \delta\left(\prod_{\ell_{*} \in \partial f_{*}} h_{\ell_{*}}\right)
$$

We will derive this result from a GFT and this will serve as a motivation to investigate GFT models as candidates for quantum gravity. Before going into that, let us recall that (2.10) is also the starting point to derive the correspondence of discretized gravity in the first order formalism with spin foams. All what is needed is the decomposition of the delta function on the group in terms of irreducible representations, labelled by the half-integer $j$

$$
\delta(h)=\sum_{j}(2 j+1) \operatorname{Tr}_{j} R^{j}(h)
$$

where $\operatorname{Tr}_{j}$ is a shorthand for $\operatorname{Tr}_{V_{j}}$, the trace in the finite dimensional vector space $V_{j}$, base of the representation. Replacing into (2.10) and decomposing the representation of the product of group elements into a product of representations we finally get

$$
Z\left[T_{*}\right]=\prod_{f_{*}} \sum_{f_{f *}}\left(2 j_{f_{*}}+1\right) \prod_{v_{*}}\{6 j\}=A_{\mathscr{G}}
$$

where all group integrations have been performed. $\{6 j\}$ are the Racah-Wigner $6 \mathrm{j}$ symbols. See for example [1] for normalization conventions. As indicated, this is exactly the spin-foam amplitude $A_{\mathscr{G}}$ of the (dual) graph $\mathscr{G}$. It represents then the transition from a given space geometry to another, as a sum over histories connecting the two.

\section{Group field theory}

In this section we introduce the basic tools of Group Field Theory (GFT) in any dimensions. GFT has been first introduced in [1, 2], then further studied by [3, 4], to describe discretized models of Quantum Gravity in terms of fields living on the dual lattice of the discretization. We first introduce the main ingredients of the theory, such as the notion of field on the group manifold, the propagator and the interaction and then we motivate our definitions showing that the quantum field theory so defined generates, in a different basis, the spin foam amplitudes of gravity in $2+1$ dimensions.

In our approach all the dynamical content of the models considered is encoded in the propagator, while the interaction vertex is fixed by the request that it be the simplest generalization of local vertices of quantum field theory.

We work with Riemannian space-times, therefore the relevant Lie group will be $S O(D)$. In three dimensions we will work with its double covering $S U(2)$. In four dimensions we will use the splitting $S O(4) \simeq S U(2) \times S U(2)$. In GFT the field arguments live on products of Lie groups

$$
\phi:\left(g_{1}, \ldots g_{D}\right) \in[S O(D)]^{D} \rightarrow \phi\left(g_{1}, \ldots g_{D}\right)
$$

with $D$ the spacetime dimension. The propagator is an Hermitean map $C: \phi \rightarrow C \phi$ with Hermitian kernel $C\left(g_{1}, \ldots, g_{D} ; g_{1}^{\prime}, \ldots, g_{D}^{\prime}\right)$ :

$$
[C \phi]\left(g_{1}, \ldots, g_{D}\right)=\int d g_{1}^{\prime} \ldots d g_{D}^{\prime} C\left(g_{1}, \ldots, g_{D} ; g_{1}^{\prime}, \ldots, g_{D}^{\prime}\right) \phi\left(g_{1}^{\prime}, \ldots, g_{D}^{\prime}\right) .
$$




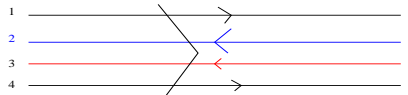

Figure 1: A stranded propagator in $\mathrm{D}=4$ with particular orientation; two strands have $\eta_{\ell f}=1$ and the other two have $\eta_{\ell f}=-1$.

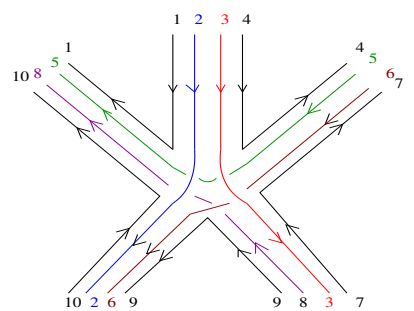

Figure 2: A simple vertex of a 4-dimensional GFT. We have chosen here a particular matching and orientation for each of the strands.

It is therefore represented by a stranded line with $D$ strands (fig. 1). The precise form of $C$ is what distinguishes the different models. It encodes the dynamical content of the theory. Vertices instead only depend on space-time dimensions and are the same for all models. We define a simple vertex joining $2 p$ strands in terms of its kernel: this is a product of $p$ delta functions matching strand arguments, so that each delta function joins two strands in two different lines. The usual vertex for $D$-dimensional GFT is a $\phi^{D+1}$ simple vertex (see fig 2). For instance the $S U(2)$ BF vertex in 3 dimensions is (with $p=6$ )

$$
S_{\text {int }}[\phi]=\frac{\lambda}{4} \int\left(\prod_{i=1}^{12} d g_{i}\right) \phi\left(g_{1}, g_{2}, g_{3}\right) \phi\left(g_{4}, g_{5}, g_{6}\right) \phi\left(g_{7}, g_{8}, g_{9}\right) \phi\left(g_{10}, g_{11}, g_{12}\right) K\left(g_{1}, . ., g_{12}\right) \text {, }
$$

with

$$
K\left(g_{1}, . . g_{12}\right)=\boldsymbol{\delta}\left(g_{3} g_{4}^{-1}\right) \boldsymbol{\delta}\left(g_{2} g_{8}^{-1}\right) \boldsymbol{\delta}\left(g_{6} g_{7}^{-1}\right) \boldsymbol{\delta}\left(g_{9} g_{10}^{-1}\right) \boldsymbol{\delta}\left(g_{5} g_{11}^{-1}\right) \boldsymbol{\delta}\left(g_{1} g_{12}^{-1}\right)
$$

A stranded graph (see fig. 3) is called regular if it has no tadpoles (hence any line $\ell$ joins two distinct vertices) and no tadfaces (hence each face $f$ goes at most once through any line of the graph). Although final results do not depend on them, it is convenient to introduce orientations in terms of incidence matrices:

- the ordinary incidence matrix $\varepsilon_{\ell v}$ which has value $1(-1)$ if the edge $\ell$ enters (exits) the vertex $v, 0$ otherwise.

- the incidence matrix $\eta_{f \ell}$ which has value +1 if the face $f$ goes through the edge $\ell$ in the same direction, -1 if the face $f$ goes through the edge $\ell$ in the opposite direction, 0 otherwise.

\section{GFT for $S O(D)$ BF theory}

In three dimensions gravity is well described by a BF theory, with gauge group $S U(2)$. The discrete partition function has been obtained in (2.10). Such a result is indeed valid for BF in any dimension. 


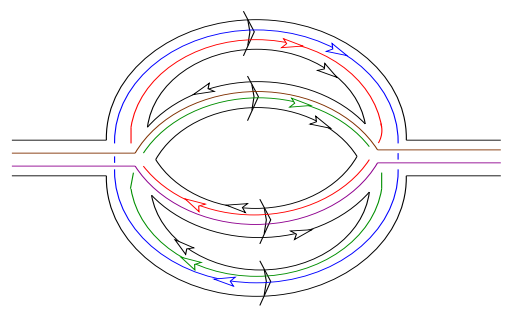

Figure 3: The "self-energy" graph $\mathscr{G}_{2}$ in 4 dimensions, quantum correction to the propagator.

We wish to reproduce such a result with a GFT model. In any dimension the propagator in direct space (that is in group variables) is just the projection on gauge invariant fields,

$$
\mathbb{P}(\phi)=\int_{S O(D)} d h \phi\left(g_{1} h, \ldots, g_{D} h\right)
$$

$\mathbb{P}^{2}=\mathbb{P}$ so that the only eigenvalues are 0 and 1 (which means that the BF theory has no dynamics). $\mathbb{P}$ is Hermitian with kernel

$$
\mathbb{P}\left(g_{1}, \ldots, g_{D} ; g_{1}^{\prime}, \ldots g_{D}^{\prime}\right)=\int d h \prod_{i=1}^{D} \delta\left(g_{i} h\left(g_{i}^{\prime}\right)^{-1}\right) .
$$

Let us look at amplitudes in direct space. We choose an arbitrary orientation of the lines and faces of a graph $\mathscr{G}$ (which for simplicity has no external legs). Combining the vertex and the propagator, integration over all strand variables $g, g^{\prime}$ leads to

$$
A_{\mathscr{G}}=\int \prod_{\ell \in L_{\mathscr{G}}} d h_{\ell} \prod_{f \in F_{\mathscr{G}}} \delta\left(\vec{\prod}_{\ell \in f} h_{\ell}^{\eta_{\ell f}}\right) .
$$

where $L_{\mathscr{G}}, F_{\mathscr{G}}$ are the set of lines and faces of $\mathscr{G}$, respectively. The oriented product $\vec{\prod}_{l \in f} h^{\eta_{\ell f}}$ means that the product of the variables $h_{\ell}$ has to be taken in the cyclic ordering corresponding to the face orientation (starting anywhere on the cycle). As announced, the amplitude (4.3) neither depends on the arbitrary orientation of the lines, nor on those of the faces. This result is valid for any $D$. In order to compare it with our previous results in discrete gravity we have to understand the graph $\mathscr{G}$ as a graph on the dual discretization of space-time. Then we can check that this is exactly the result we have found for the partition function of discretized BF (2.10). ${ }^{1}$ Therefore the Feynmann amplitudes of GFT with propagator and vertex given respectively by (4.2) and (3.3) reproduce exactly the partition function of BF models, which also means, in a different basis, spin foam transition amplitudes.

As we will see in the next section, in $3+1$ dimensions gravity is described by a constrained BF theory. The difficulty in implementing the constraints at the discrete level translates into an analogous difficulty in proposing an unambiguous GFT model.

\section{Gravity in 3+1 dimensions: the Palatini-Holst action}

It is very well known that, as for $2+1$ dimensions, we can describe General Relativity with a first order formalism which is, at least classically, equivalent to the second order formalism of the

\footnotetext{
${ }^{1}$ We will understand all GFT graphs as living in the dual triangluation from now on.
} 
Einstein-Hilbert action. Differently from the 2+1-D case, it is not just a BF theory, but we have to implement constraints. The first order action reads

$$
S=\frac{1}{8 \pi G} \int_{M} \varepsilon_{A B C D} e^{A} \wedge e^{B} \wedge F^{C D}[\omega]
$$

where $\omega=\omega_{\mu}^{A B} d x^{\mu} \tau_{A B}$ is the connection one-form, valued in the Lie algebra of $S O(3,1)$, with generators $\tau_{A B}, A=1, . .4\left(4 \times 4\right.$ antisymmetric matrices). $F=F_{\mu \nu}^{A B} d x^{\mu} \wedge d x^{v} \tau_{A B}$ is the curvature two-form; it is Lie algebra valued. The tetrads $e=e_{\mu}^{A} d x^{\mu} v_{A}$ are vector valued one-forms such that their wedge product may be regarded as $s o(3,1)$ valued, once a trivialisation chosen $e \wedge e=e_{\mu}^{A} e_{v}^{B} d x^{\mu} \wedge d x^{v} \tau_{A B}$. Therefore, similarly to the $2+1$ dimensional case, the action is entirely described in terms of Lie algebra valued forms, the curvature $F$ and the two form $e \wedge e$, whose wedge product is a 4-form which is integrated on the manifold $M$; as for their Lie algebra dependence, on identifying respectively the generators of rotations and boosts with $J_{a}, P_{a}, a=1, . ., 3$ we can use the nondegenerate bilinear form on the algebra, $<.,\rangle_{1}$

$$
<J_{a}, P_{b}>_{1}=\delta_{a b}, \quad<J_{a}, J_{b}>_{1}=<P_{a}, P_{b}>_{1}=0
$$

to rewrite the action as

$$
S_{1}=\frac{1}{8 \pi G} \int_{M} \operatorname{Tr}_{1}(e \wedge e \wedge R)
$$

with $\operatorname{Tr}_{1}:=<, . .>_{1}$. It can be easily checked that it generates the correct equations of motion. The peculiar characteristic of the group $S O(3,1)$ and of its Euclidean version $S O(4)$ is that there is another invariant quadratic form on the Lie algebra, $\operatorname{Tr}_{2}=<., .>_{2}$, so defined

$$
<J_{a}, J_{b}>_{2}=<P_{a}, P_{b}>_{2}=\delta_{a b},<J_{a}, P_{b}>_{2}=0
$$

(actually, eq. (5.4) is the specialization to $3+1$ dimensions of a quadratic form that would exist for any $\mathrm{d}+1$ ). This allows to pair the two-forms $F$ and $e \wedge e$ in a different way so that a new action is produced

$$
S_{2}=\frac{1}{8 \pi G} \int_{M} \operatorname{Tr}_{2}(e \wedge e \wedge F)=\int_{M} e^{A} \wedge e^{B} \wedge F_{A B}[\omega]
$$

with $\operatorname{Tr}_{2}:=<, .,>_{2}$. It can be easily shown that this new term can be safely added to the gravity action (5.3) without changing the equations of motion. Although we have no classical consequences, we have to expect a different quantum theory. The full action $S_{H}=S_{1}+\frac{1}{\gamma} S_{2}$ is known as the Palatini-Holst action [10], while the real parameter $\gamma$ is the Barbero-Immirzi parameter [11, 12]. $S_{H}$ can be regarded as a constrained BF action

$$
S_{H}=\int_{M} B^{A B} \wedge F_{A B}+\phi(B)
$$

where the constraint has to implement

$$
B^{A B}=\varepsilon_{C D}^{A B} e^{C} \wedge e^{D}+\frac{1}{\gamma} e^{A} \wedge e^{B} .
$$

In the so called Plebanski formulation the constraint is of the form $\phi(B) \propto \phi_{A B C D} B^{A B} \wedge B^{C D}$. The action is therefore dependent on three variables, $B, \omega, \phi$. Variations w.r.t. to all of them essentially give back Einstein equation. 
The discretization of the $4 \mathrm{D}$ BF action $S=\int_{M} B^{A B} \wedge F_{A B}$ goes along the same lines as the 3D case. We have a triangulation $\mathrm{T}$ of the space-time manifold with a 4-D simplicial complex, and a dual lattice $T^{*}$ with its 2-dimensional subcomplex which identifies our graphs. The $B$ field now being a 2 -form, has to be integrated over faces $f$ to produce $s o(4)$ (Lie algebra) elements, $B_{f}$. The discretized curvature is defined as in $3 \mathrm{D}$ to be the holonomy of the connection along the boundary of $f_{*}$ (2.7). Dual faces are now associated to faces of the direct triangulation, therefore we have:

$$
S\left(B_{f}, H_{f}\right)=\sum_{f \in T} \operatorname{Tr} B_{f} H_{f}
$$

When writing the partition function for such a discretized action, upon integrating over the Lie algebra, we end up with the same result as in (2.10) with the gauge group now $S U(2) \times S U(2)$. When using the Peter Weyl theorem to decompose the group delta function on irreducible representations, we obtain a spin foam amplitude which is the analogue of (2.12), with $6 \mathrm{j}$ symbols now replaced by $15 \mathrm{j}$ symbols (see [2] for normalization conventions) and $j \equiv\left(j_{+}, j_{-}\right)$, according to the splitting of $S O(4)$

To treat the constrained action (5.6) we have to include the Plebanski constraint. There are various proposals on how to implement it at the discrete level. We have considered just one of them, the EPRL spin-foam model [8], in the Freidel-Krasnov formulation [7], which uses coherent states. This is the argument of the next section.

\section{GFT approach to the EPRL-FK model}

In our approach all the dynamical content of different models is encoded in the propagator, while the vertex remains the same as in BF theory (3.3). Therefore the constraint is directly imposed on the propagator (4.2). This is better understood in the coherent states basis. In 4 dimensions we use the splitting of $S O(4)$ as the product $S U(2) \times S U(2)$ and we decompose the group elements as $g=\left(g_{+}, g_{-}\right)$, with $g_{ \pm} \in S U(2)$. In 3D we use $S U(2)$ coherent states $|j, g>\equiv g| j, j>=\sum_{m} \mid j, m>\left[R^{(j)}\right]_{j}^{m}(g)$. with $R^{(j)}(g)$ the $2 \mathrm{j}+1$ dimensional irreducible representation of $\mathrm{g}$. The identity is decomposed over coherent states as

$$
1_{j}=\mathrm{d}_{j} \int_{\mathrm{SU}(2)} d g|j, g><j, g|=\mathrm{d}_{j} \int_{G / H=S^{2}} d n|j, n><j, n|
$$

with $\left|j, n>=R^{(j)}\left(g_{n}\right)\right| j, j>$. In 4 dimensions we indicate with $j \equiv\left(j_{+}, j_{-}\right)$the eigenvalues of the Lie algebra generators $J$ in each $S U(2)$ component and we work with $S U(2) \times S U(2)$ coherent states $\left|j_{+}, n_{+}>\otimes\right| j_{-}, n_{-}>$. The identity is therefore

$$
1_{j}=\mathrm{d}_{j_{+}} \mathrm{d}_{j_{-}} \int_{S^{2} \times S^{2}} d n_{+} d n_{-}\left|j_{+}, n_{+}>\otimes\right| j_{-}, n_{-}><j_{+}, n_{+}\left|\otimes<j_{-}, n_{-}\right|
$$

As an intermediate step towards the full 4-dimensional case we rewrite the D-dimensional BF propagator (4.2) in a different way $(D=3$, respectively $D=4)$. Since $\mathbb{P}^{2}=\mathbb{P}$ we introduce two gaugeaveraging variables, $u, v \in G$ at the ends of the propagator, ( $u$ on the side where $\varepsilon_{v \ell}=-1$ and $v$ on the side where $\left.\varepsilon_{v \ell}=+1\right)$. G is either $S U(2)$ or $S U(2) \times S U(2)$ depending on the dimension. Between these two variables we insert the partition of unity (6.1) (resp. (6.2))

$$
\mathbb{P}\left(g ; g^{\prime}\right)=\int d u d v \prod_{f=1}^{4} \sum_{j_{f}} \mathrm{~d}_{j_{f}} \operatorname{Tr}_{V_{j_{f}}}\left(u g_{f}\left(g_{f}^{\prime}\right)^{-1} v^{-1} 1_{j_{f}}\right)
$$


where, to simplify the notation, we omit to indicate the representation explicitly and use $g \equiv R^{(j)}(g)$ for all group elements, from now on. This is equivalent to (4.2) upon redefining $h=v^{-1} u$. The index $f$ labels the strands of the propagator according to the face to which they belong. In other words, on introducing

$$
\mathbb{I}=\oplus_{j_{f}} \otimes_{f=1}^{4} 1_{j_{f}}
$$

we have used

$$
\mathbb{P}=\mathbb{P} \mathbb{P}
$$

This redundancy will be useful in a while to better understand the constrained propagator.

The amplitude of a given graph $\mathscr{G}$ with a certain number of vertices and propagators may be written as a factorization over faces

$$
A \mathscr{G}=\int \prod_{\ell \in L_{\mathscr{G}}} d u_{\ell} d v_{\ell} \prod_{f \in \mathscr{F}_{\mathscr{G}}} \mathscr{A}_{f}
$$

We assume for simplicity that all faces are closed. Then, for each given face $f$ of length $p$, we number the vertices and lines in the (anti)-cyclic order along the face as $\ell_{1}, v_{1} \cdots \ell_{p}, v_{p}$, with $\ell_{p+1}=$ $\ell_{1}$. Combining vertices (delta functions for the strand group variables, $g_{i}, g_{i}^{\prime}$ ) and propagators, and integrating over the strand group variables, $g_{i}, g_{i}^{\prime}$ we obtain

$$
\mathscr{A}_{f}=\sum_{j} d_{j}^{p+1} \prod_{a=1}^{p} \operatorname{Tr}_{j} t_{\ell_{a}, v_{a}}^{\eta_{\ell a f}} t_{\ell_{a+1, v_{a}}}^{\eta_{\ell_{a+1} f}} 1_{j}=\sum_{j} d_{j}^{p+1} \int \prod_{a=1}^{p} d n_{\ell_{a} f}<j, n_{\ell_{a} f}\left|t_{\ell_{a}, v_{a}}^{\eta_{\ell_{a f}}} t_{\ell_{a+1, v_{a}}}^{\eta_{\ell_{a+1} f}}\right| j, n_{\ell a+1, f}>
$$

where $t_{\ell_{a}, v_{a}}$ is $v_{\ell_{a}}$ if $\varepsilon_{\ell_{a} v_{a}}=+1$ and $u_{\ell_{a}}$ if $\varepsilon_{\ell_{a} v_{a}}=-1$. (Remember that in $\mathrm{D}=4 \mathrm{~d}_{j} \equiv \mathrm{d}_{j_{f+}} \mathrm{d}_{j_{f-}}$ ).

\subsection{The EPRL/FK GFT}

The EPRL/FK propagator has a structure similar to (6.3) but with replacement of $\mathbb{I}$ by a nontrivial projector which does not commute with $\mathbb{P}$. This implies that it is not possible to recombine $u$ and $v$ in a single gauge averaging variable $h$. It implements in two steps the Plebanski constraints with a non-trivial value of the Immirzi parameter $\gamma$. We only consider $0<\gamma \leq 1$ where the EPRL and FK models coincide. Starting from the expression (6.3) of the $B F$ propagator in the coherent states representation, the first step adds the constraint $j_{+} / j_{-}=(1+\gamma) /(1-\gamma), n_{+}=n_{-}$. The second step replaces in each strand of (6.3) the identity $\mathbf{1}_{j}$ by a projector $T_{j}^{\gamma}$ whose definition is

$$
T_{j}^{\gamma}=d_{j_{+}+j_{-}}\left[\delta_{j_{f-} / j_{f+}=(1-\gamma) /(1+\gamma)}\right] \int d n\left|j_{+}, n>\otimes\right| j_{-}, n><j_{+}, n\left|\otimes<j_{-}, n\right| .
$$

Grouping the four strands of a line defines an operator, $\mathbb{T}^{\gamma}$, that acts separately and independently on each strand of the propagator:

$$
\mathbb{T}^{\gamma}=\oplus_{j_{f}} \otimes_{f=1}^{4} T_{j_{f}}^{\gamma}
$$

so that the EPRL/FK propagator, to be compared with (6.5), is

$$
C=\mathbb{P} \mathbb{T}^{\gamma} \mathbb{P}
$$


that is

$$
\begin{aligned}
C\left(g, g^{\prime}\right)= & \int d u d v \prod_{f=1}^{4} \sum_{j_{f}}\left[\delta_{\left.j_{f-} / j_{f+}=(1-\gamma) /(1+\gamma)\right]} \alpha_{j_{f}} \beta_{j_{f}} \int d n_{f}\right. \\
& \operatorname{Tr}_{j_{f+} \otimes j_{f-}}\left(u g_{f}\left(g_{f}^{\prime}\right)^{-1} v^{-1}\left|j_{f+}, n_{f}>\otimes\right| j_{f-}, n_{f}\right\rangle\left\langle j_{f+}, n_{f}\left|\otimes<j_{f-}, n_{f}\right|\right),
\end{aligned}
$$

with $\alpha_{j}=d_{j_{+}} d_{j_{-}}, \beta_{j}=d_{j_{+}+j_{-}}$. We state, without proof, a number of properties of the propagator, for more details see $[9,13]$

- The operator $C$ is hermitian

- $\mathbb{T}^{\gamma}$ is a projector

- Since the propagator is hermitian, Feynman amplitudes are independent of the orientations of faces and propagators.

- Since $\mathbb{T}^{\gamma}$ and $\mathbb{P}$ do not commute, the propagator $C$ can have non-trivial spectrum (with eigenvalues between 0 and 1 ).

- Slicing the eigenvalues should allow a renormalization group analysis. This is why we call this kind of theories dynamic GFT's.

- Since $\mathbb{T}^{\gamma}$ is a projector, the propagator $C$ of the EPRL/FK theory is bounded in norm by the propagator of the $B F$ theory, as well as Feynman amplitudes.

\subsubsection{EPRL/FK Amplitudes}

Combining the propagator and the vertex expressions, the integrations over all $g, g^{\prime}$ group variables can be performed explicitly, leading to the amplitude of any graph $\mathscr{G}$. This amplitude is given by an integral of a product over all faces of the graph as in (6.6), but the amplitudes for faces are different. To compute these face amplitudes we distinguish between closed faces (no external edges) and open faces (which end on external edges). For example, the self-energy graph in fig. 3 has 6 closed faces and 4 open faces. Using the same numbering of the $p$ edges and vertices along a closed face, its amplitude is given by

$$
\mathscr{A}_{f}=\int \prod_{a=1}^{p}\left(d g_{\ell_{a}} d g_{\ell_{a}}^{\prime}\right) \sum_{j_{\ell_{a}}} \alpha_{j_{\ell_{a}}} \operatorname{Tr}_{j_{\ell_{a}}+\otimes j_{\ell_{a}}-}\left(\left(u_{\ell_{a}} g_{\ell_{a}}\left(g_{\ell_{a}}^{\prime}\right)^{-1} v_{\ell_{a}}^{-1}\right)^{\eta_{\ell_{a} f}} T_{j_{\ell_{a}}}^{\gamma}\right) \prod_{v} V_{v}
$$

where the constraint on $j_{+}, j_{-}$is implicitly understood from now on and $V_{v}$ are the vertices. To perform the integrals over the strand variables $g, g^{\prime}$ we use the standard results

$$
\begin{aligned}
& \int d g \bar{R}_{n}^{(j)}{ }_{n}^{m}(g) R^{\left(j^{\prime}\right) p}{ }_{q}(g)=\frac{1}{d_{j}} \delta\left(j, j^{\prime}\right) \delta_{q}^{m} \delta_{n}^{p} \\
& \int d g R^{(j) m}{ }_{n}(g) R^{\left(j^{\prime}\right) p}{ }_{q}(g)=\frac{1}{d_{j}} \delta\left(j, j^{\prime}\right) \varepsilon^{m p} \varepsilon_{n q}
\end{aligned}
$$


with $R^{(j) m}{ }_{n}(g)$ the matrix element of $g$ in the $2 j+1$ dimensional representation and $\bar{R}^{(j)}{ }_{n}^{m}(g)=$ $R^{(j) m}{ }_{n}\left(g^{-1}\right)$ which imply

$$
\begin{aligned}
\int d g \operatorname{Tr}_{j} A g \operatorname{Tr}_{j^{\prime}} B g^{-1} & =\frac{1}{d_{j}} \delta\left(j, j^{\prime}\right) \operatorname{Tr}_{j} A B \\
\int d g \operatorname{Tr}_{j} A g \operatorname{Tr}_{j^{\prime}} B g & =\frac{1}{d_{j}} \delta\left(j, j^{\prime}\right) \operatorname{Tr}_{j} A \varepsilon B^{T} \varepsilon^{T}
\end{aligned}
$$

and $\varepsilon \in S U(2)$,

$$
\varepsilon=\left(\begin{array}{cc}
0 & 1 \\
-1 & 0
\end{array}\right)
$$

Thus we arrive at

$$
\mathscr{A}_{f}=\sum_{j_{f}} \alpha_{j_{f}} \operatorname{Tr}_{j_{f+} \otimes j_{f-}} \vec{\prod}_{a=1}^{p}\left(h_{\ell_{a}, v_{a}}^{\eta_{\ell_{a f}}} h_{\ell_{a+1}, v_{a}}^{\eta_{\ell_{a+1} f}} T_{j_{f}}^{\gamma}\right)
$$

with $\ell_{p+1}=\ell_{1}$. It is interesting to notice that we recover the $S U(2)$ BF theory in the limit $\gamma \rightarrow 1$ (see [9] for details). For open faces (which end on external edges) we obtain a slightly modified expression but we also find that their contribution is irrelevant in the large spin approximation. It can be shown that the graph amplitude obtained by replacing (6.18) in (6.6) is the same as the spin foam amplitude found in [8] when passing to spin representation.

\section{The stationary phase method}

In [9] we have evaluated the degree of divergence of the graphs for GFT models using the stationary phase method. Here we give a brief account of the calculation, which is however quite technical, and we state the results. We refer to the original paper for more details. To this analysis, we have to rewrite the graph amplitude (6.6), with face amplitudes depending on the model, in the form

$$
\mathscr{A}_{\mathscr{G}}=\sum_{j_{f} \leq \Lambda} \mathscr{N} \int \prod d h \prod d n \exp \left\{\sum_{f} j_{f} S_{f}[h, n]\right\},
$$

with $\mathscr{N}=\mathscr{N}(j)$ and $\Lambda$ some ultraspin cutoff. To evaluate the superficial power counting, we set $j_{f}=j k_{f}$ with $k_{f} \in[0,1]$ and use the stationary phase method to derive the large $j$ behavior of the summands. This calculation has been performed both for BF models and for the EPRL/FK model. If the action is complex but has a negative real part, the contribution to this integral are quadratic fluctuations around zeroes of the real part of $S$ which are stationary points of its imaginary part, otherwise the integral is exponentially suppressed as $j \rightarrow \infty$.

\subsection{The saddle point method for 3D BF models}

For 3D BF models the degree of divergence of the graphs has already been calculated in the literature $[15,16]$. We re-derive such results to test our method. To write the action $S_{f}$ we introduce the projector

$$
|n\rangle\langle n|=\frac{1}{2}(1+\sigma \cdot n),
$$

with $|n\rangle \equiv\left|n, \frac{1}{2}\right\rangle$, and observe that

$$
\left\langle n, j|g| n^{\prime}, j\right\rangle=\left\langle n|g| n^{\prime}\right\rangle^{2 j}
$$


so that

$$
S_{f}[h, n]=k_{f} \log \operatorname{Tr}\left[\left(\prod_{\ell \in \partial f} h_{\ell}^{\eta_{\ell, f}}\right)(1+\sigma \cdot n)\right] .
$$

Since the action is the logarithm of the trace of the product of a unitary element and a projector, its real part is negative and maximal at $h_{\ell}=1$. To perform the saddle point expansion, we expand the group element to second order as

$$
h_{\ell}=1-\frac{A_{\ell}^{2}}{2}+\mathrm{i} A_{\ell} \cdot \sigma+O\left(A_{\ell}^{3}\right)
$$

with $A \in \operatorname{su}(2)$. Also

$$
n_{f}=n_{f}^{(0)}+\xi_{f}-\frac{\xi_{f}^{2}}{2} n_{f}^{(0)}+O\left(\xi_{f}^{3}\right), \quad \text { with } \quad n_{f}^{(0)} \cdot \xi_{f}=0
$$

(because $n_{f}^{2}=1$ up to third order terms). Let us consider a face with edges $\ell_{1}, \ldots, \ell_{p}$, then to second order

$$
\overrightarrow{\prod_{\ell \in \partial f}} h_{\ell}^{\eta_{\ell, f}}=1-\frac{A_{f}^{2}}{2}+\mathrm{i} \sigma \cdot A_{f}-\mathrm{i} \sigma \cdot \Phi_{f}
$$

with

$$
A_{f}=\sum_{1 \leq a \leq p} \eta_{\ell_{a}, f} A_{\ell_{a}} \quad \text { and } \quad \Phi_{f}=\sum_{1 \leq a<b \leq p} \eta_{\ell_{a}, f} \eta_{\ell_{b}, f} A_{\ell_{a}} \wedge A_{\ell_{b}}
$$

this implies

$$
S_{f}\left[A_{\ell}, \xi_{f}\right]=2 k_{f}\left\{\mathrm{i} n_{f} \cdot A_{f}-\frac{A_{f}^{2}}{2}+\frac{\left(n_{f} \cdot A_{f}\right)^{2}}{2}+\mathrm{i} \xi_{f} \cdot A_{f}+\mathrm{i} n_{f} \cdot \Phi_{f}\right\}
$$

and we have to estimate

$$
\int \prod_{\ell} d A_{\ell} \prod_{f} d \xi_{f} \exp 2 j \sum_{f} k_{f}\left\{\mathrm{i} n_{f} \cdot A_{f}-\frac{A_{f}^{2}}{2}+\frac{\left(n_{f} \cdot A_{f}\right)^{2}}{2}+\mathrm{i} \xi_{f} \cdot A_{f}+\mathrm{i} n_{f} \cdot \Phi_{f}\right\}
$$

as $j \rightarrow \infty$.

We do not integrate over the vectors $n_{f}$. They have to be chosen so that they are extrema of the imaginary part of $S$.

Because all terms except the first one $\sum_{f} k_{f} n_{f} \cdot A_{f}$ are of second order, the imaginary part is stationary if and only if

$$
\sum_{f} \mathrm{i} k_{f} n_{f} \cdot A_{f}=\sum_{\ell, f} \mathrm{i} \eta_{\ell, f} k_{f} n_{f} \cdot A_{\ell}=0 \quad \forall A_{\ell} \in \mathbb{R}^{3},
$$

which amounts to the closure condition

$$
\sum_{f} \eta_{\ell, f} k_{f} n_{f}=0, \quad \forall \ell
$$

This is the requirement that, in the semi-classical limit, the vectors $j_{f} n_{f}$ are the sides of a triangle (resp. the area bivectors of a tetrahedron) that propagates along $\ell$ in $D=3$ (resp. $D=4$ ). 
The solutions of the closure condition (7.12) range from non degenerate to maximally degenerate. In three dimensional (resp. four dimensional ) BF theory, a solution is said to be non degenerate if all the tetrahedra (resp. 4-simplices) corresponding to the vertices of the graph have maximal dimension. At the opposite end, we say that a solution is maximally degenerate if all the vectors $n_{f}$ are proportional to a single one $n_{0}$,

$$
n_{f}=\sigma_{f} n_{0} \quad \text { with } \quad \sigma_{f} \in\{-1,+1\} .
$$

In both cases we find

$$
\mathscr{A} \mathscr{G} \sim \Lambda^{3 F-3 r}
$$

with $r$ the rank of the $L \times F$ incidence matrix $\eta_{\ell, f}$.

In particular for the self-energy graph in fig. (3) we find

$$
\mathscr{A}_{\mathscr{G}_{2}} \sim \Lambda^{9}
$$

with $F=6$, the number of closed faces. We do not include open faces because they do not contribute to the divergence of the graph, as we will argue below.

\subsection{The self-energy in the EPRL/FK model}

Let us come to the EPRL/FK model. An analysis for generic graphs is possible although technically difficult. Here we only consider the self-energy graph $\mathscr{G}_{2}$ of fig. (3), which represents the first quantum correction to the propagator. Moreover, we limit the analysis to non-degenerate configurations, as in [14]. For completely degenerate configurations the stationary phase method only provides upper bounds. The issue is discussed in [9].

The self-energy graph $\mathscr{G}_{2}$ has 4 open faces and 6 closed faces with two edges. We label the internal propagators with an index $a$ ranging from 1 to 4 and orient them in the same direction. We label the 6 closed faces with pairs of indices $(a, b), a<b$. We do not worry about open faces in our analysis, which technically amounts to put to zero the external spins. However, the result should also hold for finite non-zero spins on the external faces. Indeed, since the latter remain finite, the contribution of the external faces to the action can be neglected as $j \rightarrow \infty$. The amplitude of the self-energy graph reads then

$$
\mathscr{A}_{G_{2}}=\prod_{a} d u_{a}^{ \pm} d v_{a}{ }^{ \pm} \prod_{a<b} \mathscr{A}_{a b}
$$

where, from (6.18) the face amplitude reads

$$
\begin{aligned}
\mathscr{A}_{a b} & =\sum_{j} d_{j_{+}} d_{j_{-}} \beta_{j}^{2} \int d n_{a b} d n_{a b}^{\prime}\left\langle j_{+} n_{a b}\left|u_{a+} u_{b+}^{-1}\right| j_{+} n_{a b}^{\prime}\right\rangle\left\langle j_{+} n_{a b}^{\prime}\left|v_{b+} v_{a+}^{-1}\right| j_{+} n_{a b}\right\rangle \\
& \times\left\langle j_{-} n_{a b}\left|u_{a-} u_{b-}^{-1}\right| j_{-} n_{a b}^{\prime}\right\rangle\left\langle j_{-} n_{a b}^{\prime}\left|v_{b-} v_{a-}^{-1}\right| j_{-} n_{a b}\right\rangle .
\end{aligned}
$$

In order to perform a stationary phase analysis we rewrite the graph amplitude as

$$
\mathscr{A}_{\mathscr{G}_{2}}=\sum_{j_{f}} \int \prod_{a} d u_{a}^{ \pm} \prod_{a} d v_{a}^{ \pm} \prod_{i} d n_{i} \prod_{f}\left\{\left(d_{j_{f}}\right)^{2} d_{j_{f}^{+}} d_{j_{f}^{-}} \exp \left\{j S_{f}^{+}+j S_{f}^{-}\right\}\right\}
$$


with $j_{f}^{ \pm}=j \gamma^{ \pm} k_{f}, k_{f} \in[0,1]$ and $j$ large. The face action for $f=a b$ is then

$$
S_{f}^{ \pm}=2 \gamma^{ \pm} k_{f} \log \left\{\left\langle n_{f, a}\left|u_{a}^{ \pm}\left(u_{b}^{ \pm}\right)^{-1}\right| n_{f, b}\right\rangle\left\langle n_{f, b}\left|v_{b}^{ \pm}\left(v_{a}^{ \pm}\right)^{-1}\right| n_{f, a}\right\rangle\right\}
$$

We employ the saddle point technique around the identity

$$
u_{a}^{ \pm}=1-\frac{\left(A_{a}^{ \pm}\right)^{2}}{2}+\mathrm{i} \sigma \cdot A_{a}^{ \pm}+O\left(A_{a}^{ \pm}\right)^{3}, v_{a}^{ \pm}=1-\frac{\left(B_{a}^{ \pm}\right)^{2}}{2}+\mathrm{i} \sigma \cdot B_{a}^{ \pm}+O\left(B_{a}^{ \pm}\right)^{3}
$$

and use the result (7.2) so that the action at the identity for the face $f=a b$ reads

$$
S_{f}^{ \pm}\left[1,1, n_{i}\right]=\gamma^{ \pm} k_{a b} \log \left\{\frac{1+n_{f, a} \cdot n_{f, b}}{2}\right\}
$$

which is negative except for $n_{f, a}=n_{f, b}=n_{f}$.

We perform the expansion of the coherent state around a unit vector common to all the strands of the face

$$
n_{i}=n_{f}+\xi_{i}-\frac{\left(\xi_{i}\right)^{2}}{2} n_{f}+O\left(\xi_{i}\right)^{3}, \quad \text { with } \quad n_{f} \cdot \xi_{i}=0
$$

otherwise the integral is exponentially damped. It is convenient to perform the following change of variables

$$
A_{a}^{ \pm}=A_{a} \pm \gamma^{\mp} X_{a} \quad \text { and } \quad B_{a}^{ \pm}=B_{a} \pm \gamma^{\mp} Y_{a}
$$

Terms linear in $A^{ \pm}$and $B^{ \pm}$only involve $A$ and $B$, while in the quadratic terms, the pair of variables $A$ and $B$ on one side and the pair $X$ and $Y$ on the other side decouple.

Thus we observe that, at the level of the quadratic approximation, we can separate the action into a SU(2) BF action (variables $A$ and $B$ ) and an ultralocal potential that only involves uncoupled variables attached to the vertices (variables $X$ and $Y$ ). Moreover it can be shown that this result is true for a generic graph.

We perform the Gaussian integration over the two dimensional vector $\chi_{f}=\xi_{f, a}-\xi_{f, b}$, and obtain

$$
\begin{aligned}
\mathscr{A}_{G_{2}}= & \sum_{j_{f}} j^{18}\left\{\int \prod_{a} d A_{a} \prod_{a} d B_{a} \prod_{f} d \xi_{f} \exp j S_{B F}(A, B, \xi)\right. \\
& \left.\times \int \prod_{a} d X_{a} \exp j Q(X) \times \int \prod_{a} d Y_{a} \exp j Q(Y)\right\}
\end{aligned}
$$

with $\xi_{f}=\xi_{f, a}+\xi_{f, b}$. The BF-like action is

$$
\begin{aligned}
S_{B F}[A, B, \xi]= & \sum_{a<b} k_{a b}\left\{-\frac{1}{2}\left[n_{f} \wedge\left(A_{a}-A_{b}+B_{b}-B_{a}\right)\right]^{2}\right. \\
& +\mathrm{i} n_{a b} \cdot\left(A_{a}-A_{b}+B_{b}-B_{a}\right)+\mathrm{i} n_{a b} \cdot\left(A_{a} \wedge A_{b}+B_{b} \wedge B_{a}\right) \\
& \left.+\mathrm{i} \xi_{a b} \cdot\left(A_{a}-A_{b}+B_{b}-B_{a}\right)\right\}
\end{aligned}
$$

while the ultra local terms are

$$
Q[X]=\gamma^{+} \gamma^{-} \sum_{a<b} k_{a b}\left\{\left[n_{a b} \wedge\left(X_{a}-X_{b}\right)\right]^{2}+\mathrm{i} n_{a b} \cdot\left(X_{a} \wedge X_{b}\right)\right\} .
$$


The Gaussian integral over the variables $A$ and $B$ can be evaluated using the same techniques as for the pure BF models, thus yielding

$$
\int \prod_{a} d A_{a} \prod_{a} d B_{a} \prod_{f} d \xi_{f} \exp j S_{B F}(A, B, \xi) \sim j^{-9}
$$

as $j \rightarrow \infty$. As for the Gaussian integrals over the independent variables $X_{a}$ and $Y_{a}$, we have

$$
\int \prod_{a} d X_{a} \exp j Q(X) \sim j^{-\frac{\operatorname{rank}(Q)}{2}}
$$

with $Q[X]$ the quadratic form in Eq. (7.29) and a similar expression for $Q[Y]$. The rank may be computed to be $\operatorname{rank}(\mathrm{Q})=9$ so that the Gaussian integral over $X, Y$ yields a power of $j^{-9 / 2}$ each. Therefore, we obtain the power counting for the self-energy with non degenerate configurations as follows

$$
\sum_{6 \text { independent spins } \sim j<\Lambda} j^{24} \times j^{-6} \times j^{-9} \times\left(j^{-9 / 2}\right)^{2} \sim \Lambda^{6},
$$

where

- $j^{24}$ arises from a $d_{j^{+}} d_{j^{-}} \sim j^{2}$ for each of the 6 faces

- $d_{j} \sim j$ for each of the two strands in each face

- $j^{-6}$ results from the Gaussian integration over the 6 variables $\chi_{f}=\left(\xi_{f, a}-\xi_{f, b}\right)$

- $j^{-9}$ from the integration over $A$ and $B$

This reproduces the result of [PRS], with non degenerate configurations.

\section{References}

[1] D. V. Boulatov, A Model of three-dimensional lattice gravity, Mod. Phys. Lett. A7 (1992) 1629; [arXiv:hep-th/9202074].

[2] H. Ooguri, Topological lattice models in four-dimensions, Mod. Phys. Lett. A7 (1992) 2799; [arXiv:hep-th/9205090].

[3] L. Freidel, Group field theory: An overview, Int. J. Theor. Phys. 44, 1769 (2005) [arXiv:hep-th/0505016].

[4] D. Oriti, The group field theory approach to quantum gravity, [arXiv:gr-qc/0607032].

[5] J. C. Baez, An introduction to spin foam models of BF theory and quantum gravity, Lect. Notes Phys. 543, 25 (2000) [arXiv: gr-qc/9905087].

[6] J. Barrett and L. Crane Relativistic spin networks and Quantum Gravity, J. Math. Phys. 39, 3296 (1998) [arXiv:gr-qc/9709028].

[7] L. Freidel and K. Krasnov, A New Spin Foam Model for 4d Gravity, Class. Quant. Grav. 25, 125018 (2008) [arXiv:0708.1595 [gr-qc]].

[8] J. Engle, E. Livine, R. Pereira and C. Rovelli, LQG vertex with finite Immirzi parameter, Nucl. Phys. B 799, 136 (2008) [arXiv:0711.0146 [gr-qc] ]. 
[9] T. Krajewski, J. Magnen, V. Rivasseau, A. Tanasa, P.Vitale Quantum Corrections in the Group Field Theory Formulation of the EPRL/FK Models, Phys. Rev. D 82124069 (2010) [arXiv: 1007.3150 $[\mathrm{gr}-\mathrm{qC}]]$.

[10] S. Holst, Barbero's Hamiltonian derived from a generalized Hilbert-Palatini action, Phys. Rev. D 53, 5966 (1996) [arXiv: gr-qc/9511026].

[11] J. F. Barbero G., Reality conditions and Ashtekar variables: A Different perspective, Phys. Rev. D 51, 5498 (1995) [arXiv: gr-qc/9410013].

[12] G. Immirzi, Real and complex connections for canonical gravity, Class. Quant. Grav. 14, L177 (1997) [arXiv: gr-qc/9612030].

[13] J. B. Geloun, R. Gurau and V. Rivasseau, Europhys. Lett. 92 (2010) 60008 [arXiv: 1008.0354 [hep-th]].

[14] C. Perini, C. Rovelli and S. Speziale, Self-energy and vertex radiative corrections in LQG, Phys. Lett. B 682, 78 (2009) [arXiv:0810.1714 [gr-qc] ].

[15] L. Freidel, R. Gurau and D. Oriti, Group field theory renormalization - the 3 d case: power counting of divergences, Phys. Rev. D 80, 044007 (2009) [arXiv:0905.3772 [hep-th] ].

[16] J. B. Geloun, T. Krajewski, J. Magnen and V. Rivasseau, Linearized Group Field Theory and Power Counting Theorems, Class. Quant. Grav. 27 (2010) 155012 [arXiv:1002.3592 [hep-th]]. 